https://doi.org/10.46813/2021-132-069

\title{
THE LIFSHITZ-ROSENZWEIG METHOD IN CALCULATIONS OF THE BIAS OF BASIC DISLOCATION LOOPS IN ZIRCONIUM
}

\author{
O.G. Trotsenko, A.V. Babich, P.M. Ostapchuk \\ Institute of Electrophysics and Radiation Technologies of the NAS of Ukraine, \\ Kharkiv, Ukraine \\ E-mail:oleg-trotsenko@ukr.net
}

\begin{abstract}
Analytical expressions for the elastic interaction energy of radiation point defects of the dipole type with basic dislocation loops in zirconium are obtained for edge with Burgers vector $b^{D}=1 / 2[0001]$ and mixed with $b^{\mathrm{DS}}=1 / 6<20 \overline{2} 3>$ using Lifshitz-Rosenzweig method. They were used in numerical calculation (by the finite difference method) of the bias of these loops in a toroidal reservoir taking into account the elastic anisotropy of a hexagonal crystal. The toroidal geometry of the reservoir allows calculations for a loop of any size and without any correction of the elastic field in its area of influence. In the approximation of the center of dilatation, the dependences of the bias of loops on their radius and nature are obtained. It is suggested that bias is determined only by the edge component of its Burgers vector. The essential role of the form of the boundary condition on the outer surface of the reservoir is shown.
\end{abstract}

PACS: 62.20.Dc; 62.20.Fe

\section{INTRODUCTION}

It is known that radiation damage in metals and alloys during their bombardment by energy particles is manifested in the form of atomic displacements, the initial morphology of which depends on the energy transmitted during the collision of particles with lattice atoms. Thus, megaelectronvolt electrons create damage in the form of Frenkel pairs, i. e. isolated vacancies and self-interstitial atoms (SIA). Fast neutrons and heavy ions cause damage in the form of displacement cascades, the thermal relaxation of which leads to the formation of not only single Frenkel pairs, but also embryos of interstitial and vacancy dislocation loops. Further diffusion evolution of the latter plays a very important role in many phenomena of radiation-induced deformation of irradiated metals and alloys. A typical example of such deformation is the radiation growth (RG) of hexagonal close-packed (hcp) metals, in particular zirconium - the main structural material in the production of fuel elements and fuel assembls of nuclear reactors of the WWER type [1]. The RG phenomenon is accompanied by a change in the shape of the material without the application of an external load and without a noticeable change in volume. Thus, zirconium expands in $\langle a\rangle$-direction and shrinks along the $\langle c\rangle$-axe during the process of radiation growth $[2,3]$. This disturbs the stability of the initial geometry of the fuel claddings, which negatively affects the service life of the reactor. Such behavior of zirconium is associated with the nucleation and growth of vacancy dislocation loops on the basal planes, "eating" the crystal along $\langle c\rangle$-axe. At the same time, mainly interstitial loops should grow on prismatic planes, forming additional extraplanes in $\langle a\rangle$-direction. However, the mechanism for the growth of vacancy loops is not yet clear.

The most popular version of the cause of radiation growth of zirconium is anisotropic diffusion of radiation point defects (PD) between its planes (DAD theory diffusional anisotropy difference) [4,5]. Its main assumption is as follows $D_{i}^{a} / D_{i}^{c}>D_{v}^{a} / D_{v}^{c}$. Here $D_{m}^{a}$ is the diffusion coefficient of PD of $m$-type in the basal plane of zirconium; $D_{m}^{c}$ is the diffusion coefficient in $\langle c\rangle$-direction (subscript $i$ and $v$ refer to vacancies and SIA respectively). However, there is no experimental confirmation of this inequality to date. Moreover, recent numerical calculations [6] have shown that vacancies and SIA really migrate diffusionally mainly parallel to the basal plane $\left(D_{i, v}^{a} / D_{i, v}^{c}>1\right)$, however, the inequality in the range of reactor temperatures $(T<800 \mathrm{~K})$ is just the opposite $D_{i}^{a} / D_{i}^{c}<D_{v}^{a} / D_{v}^{c}$. Therefore, the classical elastic ideology: EID - elastic interaction difference [5] is used in the paper. Its central element is the concept of preferential absorption by internal extended crystal defects (sinks) of radiation PD of a certain type, known as bias. The physics is that SIA elastically interacts with dislocations more strongly than vacancies. As a result, the total diffusion flux of SIAs on them is greater than the vacancy flux, and this difference is interpreted as a preference for the absorption of SIAs. The vacancies remaining in excess are absorbed by the pores. This way is accepted to explain the phenomenon of radiation swelling of cubic metals and alloys. As for the radiation growth, within the framework of the elastic ideology, the growth of vacancy loops in zirconium is possible in the presence of an uncompensated vacancy flux into the basal plane. The latter means that prismatic interstitial loops, as well as edge dislocations, should more strongly absorb SIA, i.e. their bias should be higher than the basic ones. Therefore, when constructing a theory of $\mathrm{RG}$, one must be able to calculate this value for both types of loops. In this regard, the paper presents the methodology and results of calculating the bias of two types of basis loops in zirconium with Burgers vectors 
$b^{D}=1 / 2[0001]$ and $b^{D S}=1 / 6<20 \overline{2} 3>$. These are vacancy loops, the first is purely edge, and the second contains a shear component.

\section{LOOP BIAS. FORMULATION OF THE PROBLEM}

The sink bias is determined by a relation of the form $B=1-Z_{v} / Z_{i}$. Here subscripts $v$ and $i$ correspond to vacancies and SIA respectively. If $B>0$, one says that the loop has a preference to SIA. The dimensionless quantity $Z_{v, i}$ is called the absorption efficiency of the PD by the sink. It appears as a result of calculating the PD diffusion flux to a specific sink. Under the assumption of diffusion isotropy of the medium $\left(D_{i j}=D \delta_{i j}\right)$ the PD flux $J$ to the dislocation loop is found by solving the following diffusion problem in its region of influence using the quasi-stationary approximation:

$$
\begin{aligned}
& \omega \operatorname{div} \boldsymbol{j}(\boldsymbol{r})=0 ; \omega \boldsymbol{j}(\boldsymbol{r})=-D C(\boldsymbol{r}) \beta \nabla \mu(\boldsymbol{r}) ; \\
& \beta \equiv 1 / k_{B} T ; \\
& \beta \mu(\boldsymbol{r})=\ln \left(\frac{C(\boldsymbol{r})}{C^{e}} \exp \left(\beta E_{\mathrm{int}}(\boldsymbol{r})\right)\right) ; \\
& J=-\iint_{S}[\boldsymbol{n} \boldsymbol{j}(\boldsymbol{r})] d \sigma .
\end{aligned}
$$

Here $C(\boldsymbol{r})$ is the concentration of migrating PD; $\boldsymbol{j}(\boldsymbol{r})$, $\mu(\boldsymbol{r})$ - their flux density and chemical potential, respectively; $E_{\text {int }}(\boldsymbol{r})$ - their interaction energy with the loop; $C^{e}$ - equilibrium thermal concentration of PD in the crystal in the absence of a stress field $E_{\text {int }}$. The integral is taken over an arbitrary surface containing the loop with the outer normal $\boldsymbol{n}$. Equation (1) should be supplemented with boundary conditions. The inner surface $S_{\mathrm{C}}$ is usually chosen in the form of a torus containing a dislocation line. The torus minor radius $r_{c}$ corresponds to the dislocation core radius. The boundary condition on it has the form:

$$
\left.C(\boldsymbol{r}) \exp \left(E_{\text {int }}(\boldsymbol{r})\right)\right|_{S_{C}}=0 \text {. }
$$

The condition has the traditional form and corresponds to the value of the PD chemical potential at the dislocation core $\left.\mu\right|_{S_{C}}=0$, when we neglect the linear tension of the loop and the effect of coalescence of loops of the same nature during annealing. Outer surface $S_{e x t}$ following the authors [7] we choose in the form torus coaxial with $S_{\mathrm{C}}$ with generating circle radius $\mathrm{R}_{\mathrm{ext}}$ which corresponds to the radius of the loop influence region. By analogy with (2) we formulate it for the chemical potential in the form: $\left.\beta \mu\right|_{S_{\text {ext }}}=\ln \left(\bar{C} / C^{e}\right)$. Here $\bar{C}$ is the average PD concentration in an effective medium that simulates the influence of all sinks. This is the standard form of the PD chemical potential in an effective medium, where the influence of a particular sink is neutralized by the others. Then:

$$
\left.C(\boldsymbol{r}) \exp \left(E_{\text {int }}(\boldsymbol{r})\right)\right|_{S_{\text {ext }}}=\bar{C} .
$$

It remains to find interaction energy of PD with the loop. According to Eshelby [8] it looks like:

$$
\begin{gathered}
E_{\text {int }}(\boldsymbol{r})=-P_{i j} u_{i j}(\boldsymbol{r}) ; \\
P_{i j}=P_{j i} .
\end{gathered}
$$

Here $u_{i j}(\boldsymbol{r})$-deformation field caused by the loop in the point where the PD is situated. The point defect itself is described by the distribution of volumetric forces without moment. If the elastic dipole has an axis of symmetry which coincides with $\langle\mathrm{c}\rangle$-axis of hpc crystal, then tensor $P_{i j}$ has only diagonal components, which in the abbreviated description can be written as $P_{i}=P(1,1, \varepsilon), P=P_{a}, \varepsilon=P_{c} / P_{a}$. Here $P_{a}$ and $P_{c}-$ strength of the force-dipoles in $\langle a\rangle$ - and $\langle c\rangle$ directions. Note that $P_{a}$ and $P_{c}$ do not have a simple physical meaning for defect in crystal with non-cubic symmetry. Therefore, in papers $[9,10]$, by analogy with $P_{i}=P(1,1, \varepsilon)$, displacement dipoles $Q_{i}=Q(1,1, \delta)$, $Q=Q_{a}, \quad \delta=Q_{c} / Q_{a} \quad$ were introduced. They are connected with force-dipoles by relation $P_{i j}=C_{i j} Q_{j}$, $C_{i j}$ is the crystal elastic moduli. In this case, it was assumed that the change in the volume $\Delta V$ of the finite crystal caused by a point defect is related to the displacement dipoles by the relation $\Delta V=Q(2+\delta)$. Then for hexagonal crystal:

$$
\begin{gathered}
E_{\text {int }}(\boldsymbol{r})=-\Delta V \frac{C_{11}+C_{12}+\delta(\varepsilon) C_{13}}{2+\delta(\varepsilon)} \times \\
\times\left[S p u_{i j}(\boldsymbol{r})-(1-\varepsilon) u_{i j}(\boldsymbol{r})\right], \\
\delta(\varepsilon)=\frac{\varepsilon\left(C_{11}+C_{12}\right)-2 C_{13}}{C_{33}-\varepsilon C_{13}}
\end{gathered}
$$

and one need to calculate the deformation field created by a particular loop.

There are two ways of calculating the field $u_{i j}(\boldsymbol{r})$. First one is solving of equilibrium equations in terms of displacements with appropriate boundary conditions. In the case of zirconium, it is applicable for a loop with a Burgers vector $b^{D}=1 / 2[0001]$ (axial symmetry). The second is through the tensor Green's function $\mathrm{G}_{\mathrm{ij}}$ (TGF) equations of equilibrium of a given elastic medium, which allows calculating the displacements created by a loop with any Burgers vector. The second option is used in the work, a TGF is calculated by the method LifshitzRosenzweig [11]. As a result, for the vacancy loop $\mathrm{b}^{\mathrm{D}}=1 / 2[0001]$ and dilatation center $(\varepsilon=1)$ in the Cartesian coordinate system we have [12]: 


$$
\begin{gathered}
E_{\mathrm{int}}^{D}(\boldsymbol{r})=\Delta V P \frac{b^{D}}{4 \pi} \int_{S_{D}} \frac{d^{2} r^{\prime}}{\left|\boldsymbol{r}-\boldsymbol{r}^{\prime}\right|^{3}}\left[\left(1-3 \tau_{3}^{2}\right) Q\left(\tau_{3}^{2}\right)+2 \tau_{3}^{2}\left(1-\tau_{3}^{2}\right) \frac{d Q}{d \tau_{3}^{2}}\right] \\
P=\frac{2 C_{13}^{2}-\left(C_{11}+C_{12}\right) C_{33}}{4 C_{13}-2 C_{33}-\left(C_{11}+C_{12}\right)} \\
Q\left(\tau_{3}^{2}\right)=C_{13}\left[V\left(\tau_{3}^{2}\right)+\mathrm{K}\left(\tau_{3}^{2}\right)\right]+C_{33}\left[V\left(\tau_{3}^{2}\right)+W\left(\tau_{3}^{2}\right)\right] ; \quad \tau_{3}^{2}=z^{2} /\left|\boldsymbol{r}-\boldsymbol{r}^{\prime}\right|^{2} \\
V\left(\tau_{3}^{2}\right) \equiv\left(1-3 \tau_{3}^{2}\right) \Phi\left(\tau_{3}^{2}\right)+2 \tau_{3}^{2}\left(1-\tau_{3}^{2}\right) \frac{d \Phi}{d \tau_{3}^{2}} \\
W\left(\tau_{3}^{2}\right) \equiv F\left(\tau_{3}^{2}\right)-2\left(1-\tau_{3}^{2}\right) \frac{d F}{d \tau_{3}^{2}} \\
\mathrm{~K}\left(\tau_{3}^{2}\right) \equiv-\mathrm{N}\left(\tau_{3}^{2}\right)-2 \tau_{3}^{2} \frac{d \mathrm{~N}}{d \tau_{3}^{2}}-3 \tau_{3}^{2} \mathrm{M}\left(\tau_{3}^{2}\right)+2 \tau_{3}^{2}\left(1-\tau_{3}^{2}\right) \frac{d \mathrm{M}}{d \tau_{3}^{2}}
\end{gathered}
$$

where integration is carried out over an arbitrary surface $S_{D}$, based on the line of dislocation; $\boldsymbol{r}$ - observation point coordinate; $\boldsymbol{r}^{\prime}$ - surface point coordinate $S_{D} ; b^{D}$ is $z$-component of the Burgers vector. Functions $\Phi\left(\tau_{3}^{2}\right), \quad F\left(\tau_{3}^{2}\right), \quad \mathrm{N}\left(\tau_{3}^{2}\right), \quad$ and $\quad \mathrm{M}\left(\tau_{3}^{2}\right)$ are very complicated. Their explicit expressions are given in [12]. It is important that they all depend on only one variable $\tau_{3}^{2}$. To calculate a similar value for the loop $\mathrm{b}^{\mathrm{DS}}=1 / 6<20 \overline{2} 3>$, it is convenient to represent its Burgers vector as the sum of two vectors $1 / 6[20 \overline{2} 3]=1 / 2[0001]+1 / 3[10 \overline{1} 0] \equiv b^{D}+b^{S}$. Then, choosing the axis " $x$ " of basal plane "xy" Cartesian coordinate system along $b^{\mathrm{s}}$, we get:

$$
\begin{aligned}
& E_{\mathrm{int}}^{D S}(\boldsymbol{r})=E_{\mathrm{int}}^{D}(\boldsymbol{r})-\Delta V P \frac{b^{S}}{4 \pi} \int_{S_{D}} \frac{d^{2} r^{\prime}}{\left|\boldsymbol{r}-\boldsymbol{r}^{\prime}\right|^{3}} \tau_{3} \tau_{1} H\left(\tau_{3}^{2}\right) ; \tau_{1}=\left(x-x^{\prime}\right) /\left|\boldsymbol{r}-\boldsymbol{r}^{\prime}\right| ; \\
& H\left(\tau_{3}^{2}\right)=3\left\{\Psi\left(\tau_{3}^{2}\right)-Y\left(\tau_{3}^{2}\right)\right\}+2 \tau_{3}^{2} \frac{d \Psi}{d \tau_{3}^{2}}+2\left(1-\tau_{3}^{2}\right) \frac{d Y}{d \tau_{3}^{2}} ; \\
& \Psi\left(\tau_{3}^{2}\right)=C_{44}\left[V\left(\tau_{3}^{2}\right)+W\left(\tau_{3}^{2}\right)\right] ; \quad Y\left(\tau_{3}^{2}\right)=C_{44}\left[V\left(\tau_{3}^{2}\right)+\mathrm{K}\left(\tau_{3}^{2}\right)\right] .
\end{aligned}
$$

Note that the second term in (7), in contrast to $E_{\text {int }}^{D}$, is symmetric with respect to replacement $\mathrm{z} \rightarrow-\mathrm{z}$, $\mathrm{x} \rightarrow-\mathrm{x}$, so in the lower half-plane $(\mathrm{z}<0)$ the picture is the same, but turned to $180^{\circ}$. Thus, it is necessary to solve the diffusion problem (1)-(3) numerically in the region of influence of a loop with a given energy of its elastic interaction with PD (6), (7) and find its bias.

\section{CALCULATION PROCEDURE AND RESULTS}

We assume that the loops are in the form of a circle with a radius $\mathrm{R}$, lying in the plane $\mathrm{z}=0$ (zirconium basal plane) of a cylindrical system coordinates $(r, \varphi, z)$. All calculations are performed using dimensionless coordinates $\quad \mathrm{r} \rightarrow \mathrm{r} / \mathrm{b}^{\mathrm{D}} ; \quad \mathrm{z} \rightarrow \mathrm{z} / \mathrm{b}^{\mathrm{D}}$; $\left(x-x^{\prime}\right)=r \cos \varphi-r^{\prime} \cos \varphi^{\prime} ; \quad \mathrm{z}^{\prime}=0, \quad$ since the integration in (6), (7) is carried out over the area of the loop; $\left|\boldsymbol{r}-\boldsymbol{r}^{\prime}\right|^{2}=r^{2}+z^{2}-2 r r^{\prime} \cos \left(\varphi-\varphi^{\prime}\right)+r^{\prime 2}$. Note that due to isotropy in the crystal basal plane, the dependence on the azimuthal angle $\varphi$ in (6) is absent. At the same time, in (7) it is conserved in the second term due to the presence $\tau_{1}$ and it produces significant problems in numerical calculations. Figs. 1 and 2 for loop with radius $\mathrm{R}=20$ in plane $\mathrm{z}=3$ illustrate this dependency in (7) $\mathrm{I}^{\mathrm{LR}}(\mathrm{r}, \mathrm{z}, \varphi, \mathrm{R})=\int_{\mathrm{S}_{\mathrm{D}}} \frac{\mathrm{d}^{2} \mathrm{r}^{\prime}}{\left|\boldsymbol{r}-\boldsymbol{r}^{\prime}\right|^{3}} \tau_{3} \tau_{1} \mathrm{H}\left(\tau_{3}^{2}\right)$ for two regions: inner $r=10$ (point line), $r=17$ (solid line) (see Fig. 1) and external $r=23$ (point line), $r=25$ (solid line) (see Fig. 2) (all values are measured in $b^{\mathrm{D}}$ ). Wherein experimental values of the elastic moduli of zirconium according to [13] (Mbar) look like: $C_{11}=1.154 ; \quad C_{12}=0.672 ; \quad C_{13}=0.646 ; \quad C_{33}=1.725 ;$ $C_{55}=C_{44}=0.363$. One can see, that in both regions the function $\mathrm{I}^{\mathrm{LR}}(\varphi)$ changes sign when $\varphi=\pi / 2$ and $\varphi=3 \pi / 2$, and in absolute value it increases with approaching the dislocation line. It should be said that the bias is not experimentally determined. It is estimated 
theoretically and a prediction is made regarding the macroscopic deformation of the material, which can be observed and measured experimentally. Therefore, in order to avoid unnecessary complications in the numerical calculation, we exclude the dependence on the azimuthal angle $\varphi$, averaging over it the function

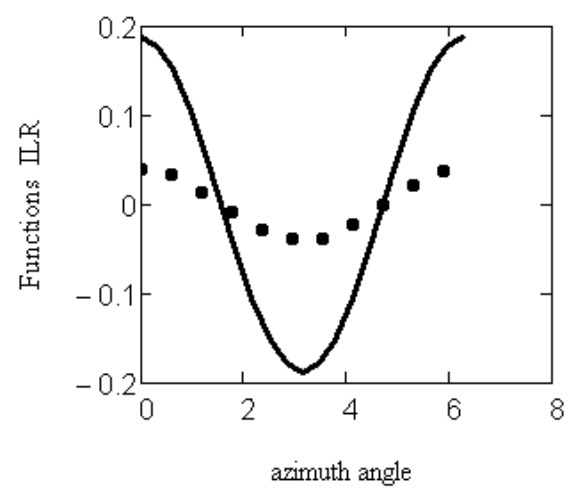

Fig. 1. Dependency of $I^{L R}(\varphi)$ on the azimuth angle for $R=20 ; z=3$ and inner region of the loop $r=10$ ( point line), $r=17$ (solid line)

In terms of $\quad$ a $\quad$ variable $\psi(r, z)=C(r, z) \exp E_{\text {int }}(r, z) / \bar{C}$ the diffusion problem (1)-(3) in dimensionless cylindrical coordinates taking into account isotropy in the basal plane has the form:

$$
\frac{\partial^{2} \psi}{\partial r^{2}}+\frac{\partial^{2} \psi}{\partial z^{2}}+\left(\frac{1}{r}-\frac{\partial E_{\text {int }}}{\partial r}\right) \frac{\partial \psi}{\partial r}-\frac{\partial E_{\text {int }}}{\partial z} \frac{\partial \psi}{\partial z}=0
$$

with boundary conditions

$$
\begin{gathered}
\psi(r, z)=0 \quad \text { on the inner toroidal surface } \\
R-r_{c} \leq r \leq R+r_{c} ; \\
\psi(r, z)=1 \quad \text { on the outer toroidal surface } \\
R-R_{e x t} \leq r \leq R+R_{e x t} \quad \text { for } \quad R>R_{e x t} ;
\end{gathered}
$$

$\mathrm{I}^{\mathrm{LR}}(\varphi)$. Thus, in one half of the loop $(3 \pi / 2 \leq \varphi \leq 5 \pi / 2)$ this will be a positive addition to $E_{\text {int }}^{D}$ in (7), and negative in another $(\pi / 2 \leq \varphi \leq 3 \pi / 2)$.

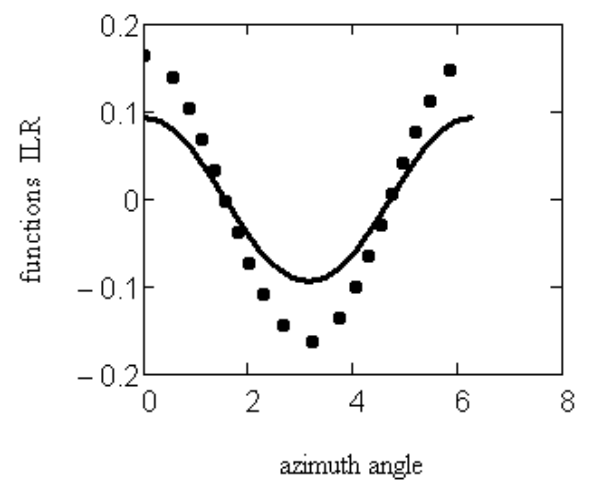

Fig. 2. Dependency of $I^{L R}(\varphi)$ on the azimuth angle

for $R=20 ; z=3$ and outer region of the loop $r=23$ ( point line), $r=25$ (solid line)

$$
0 \leq r \leq R+R_{e x t} \text { for } R<R_{e x t}
$$

Then for the flux and absorption efficiency $Z_{v, i}$ we have:

$$
\begin{gathered}
J=2 \pi R \frac{D \bar{C}}{\omega} Z\left(r_{c}, R, R_{e x t}\right), \\
Z\left(r_{c}, R, R_{e x t}\right)=\frac{1}{2 \pi R} \iint_{S} \exp \left(-E_{\text {int }}(r, z)\right) \times \\
\times[n \nabla \psi(r, z)] d \sigma .
\end{gathered}
$$

The diffusion problem (8), (9) was solved numerically by the finite difference method. Fig. 3 shows a cross section of a toroidal reservoir containing a loop [14].
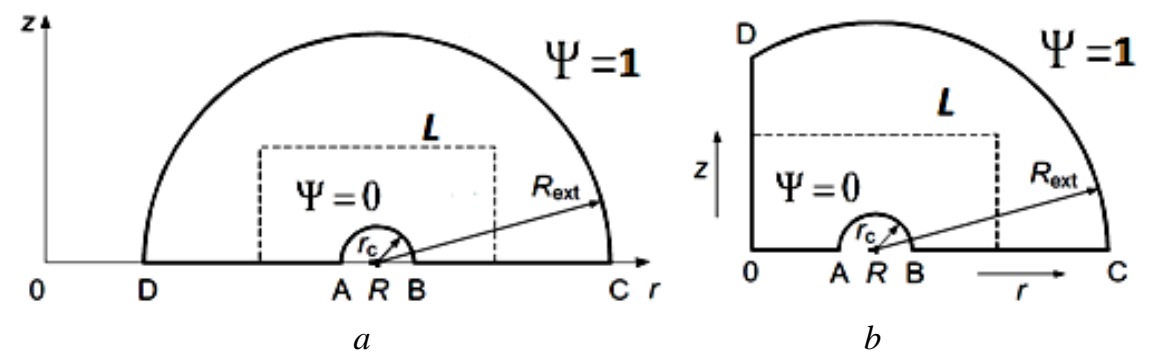

Fig. 3. Coordinate system for a toroidal reservoir: $a-R>R_{\text {ext }} ; b-R<R_{\text {ext }}$

It is important to note that in the event (6) $\left(b^{D}=1 / 2[0001]\right)$ the expression for the interaction energy of the loop with the PD is symmetric with respect to rotation around the "z" axis (does not depend on the azimuthal angle $\varphi$ ) and to change of variables $\mathrm{z} \rightarrow-\mathrm{z}$. So its bias is determined by one capture efficiency PD of type $m\left(Z_{m}\right) B^{D}=1-Z_{v} / Z_{i}$. In case (7) $\left(b^{\mathrm{DS}}=1 / 6<20 \overline{2} 3>\right)$, although the average addition to $E_{\text {int }}^{D}$ does not depend on $\varphi$, but it is of the opposite sign in different halves of the loop. Therefore, the value of its bias is determined by two efficiencies $\left(Z_{m}^{( \pm)}\right)$ $B^{D S}=1-\left(Z_{v}^{(+)}+Z_{v}^{(-)}\right) /\left(Z_{i}^{(+)}+Z_{i}^{(-)}\right)$, corresponding to positive (+) and negative (-) additions to (7).

Fig. 4 shows the dependences of the bias of the basis loops of zirconium $\left(B^{D}\right.$ and $B^{D S}$ ) on their radius (in $b^{D}$ ) for two values of the outer size of the toroidal 
reservoir: $R_{\text {ext }}=200 b^{D}$, which corresponds to the dislocation density $\rho \approx 8 \cdot 10^{9} \mathrm{~cm}^{-2}$ (see Fig. 4,a) and $R_{\text {ext }}=90 b^{D} \quad\left(\rho \approx 4 \cdot 10^{10} \mathrm{~cm}^{-2}\right.$ (see Fig. 4,b)). Curves with a maximums refer to vacancy loops, with a minimum to interstitial; "•", “ $\boldsymbol{\Delta}$ " correspond to loops with $b^{D}=1 / 2[0001]\left(B^{D}\right)$, solid lines to loops with

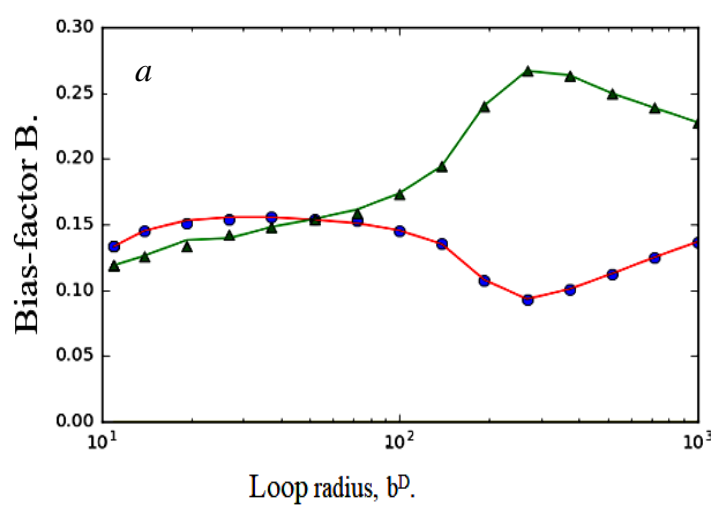

$\mathrm{b}^{\mathrm{DS}}=1 / 6<20 \overline{2} 3>\left(B^{D S}\right)$. Good quality agreement $B^{D}$ with $B^{D S}$ is seen (we remind that in the framework of the approximation associated with averaging over the azimuthal angle). Hence follows an interesting and, possibly, rather general conclusion that the bias of a loop is determined only by the edge component of its Burgers vector.

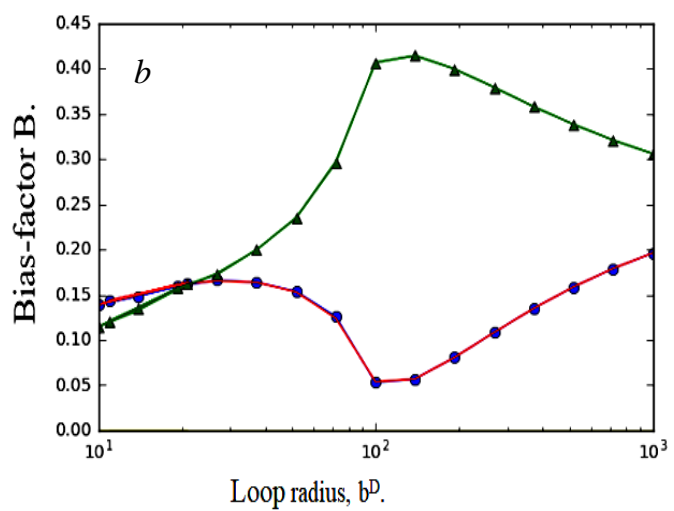

Fig. 4. Dependences of the bias of the basis loops of zirconium $\left(B^{D}\right.$ and $\left.B^{D S}\right)$ on their radius for two values of the external size of the toroidal reservoir: $a-R_{\text {ext }}=200 b^{D} ; b-R_{\text {ext }}=90 b^{D}$

It should also be noted that, in the region of small sizes, vacancy loops have the smallest bias compared to interstitial loops and rectilinear dislocations; they are the main sink for vacancies, which contributes to their

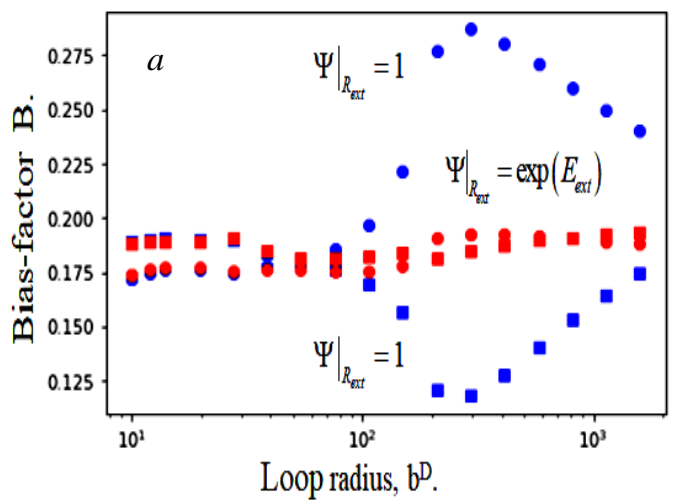

nucleation. In the region of large sizes, the situation is the opposite. Interstitial loops become the main sinks for vacancies. Therefore, they have no chance of survival, which is observed in experiments.

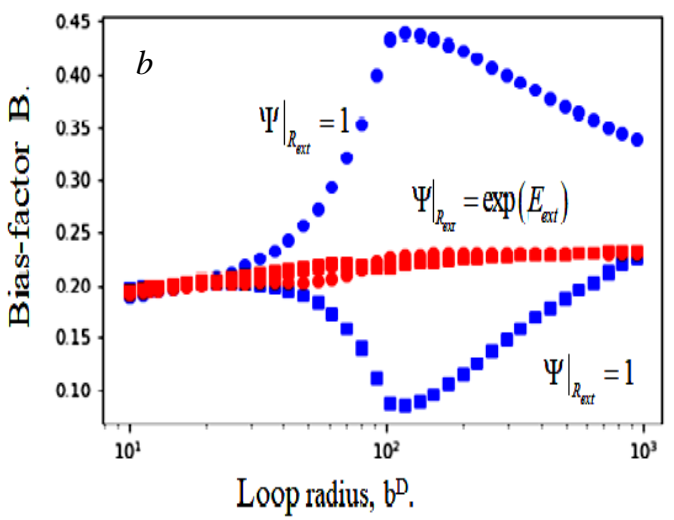

Fig. 5. Dependences of the bias of the loop with $b^{D}=1 / 2[0001]\left(B^{D}\right)$ with different boundary conditions on the outer radius of the toroidal reservoir: $a-R_{\text {ext }}=200 b^{D} ; b-R_{e x t}=90 b^{D}$.

- - corresponds to the vacancy loop; - to the interstitial

Fig. 5 reflects the role of the type of boundary condition on the outer radius of the toroidal reservoir $\mathrm{R}_{\text {ext }}$. In our case (9) it is $\psi(r, z)=1$, in paper [7] it is $\psi=\exp E_{\text {int }}$. The result [7] is shown in Fig. 5 in red for a loop with $\mathrm{b}^{\mathrm{D}}=1 / 2[0001] \quad\left(B^{D}\right)$ in case $R_{\text {ext }}=200 b^{D} \quad$ (see Fig. 5,a) and $R_{\text {ext }}=90 b^{D}$ (see Fig. 5,b): dependence of the bias on the loop radius does not depend on its nature (vacancy or interstitial). The result of solution (8), (9) is shown in blue. It is seen that in the region of small loop sizes bias does not depaend on kind of boundary condition on the outer radius of the toroidal reservoir $\mathrm{R}_{\mathrm{ext}}$. With an increase in the size of the loops, this symmetry is broken. In case $\psi=\exp E_{\text {int }}$
[7] we see monotonic growth of bias, in our case (9), a maximum appears for vacancy loops, and a minimum for interstitial loops. However, with $\mathrm{R} \rightarrow \infty$ both dependencies go to the same constant value of bias corresponding to rectilinear dislocation. This behavior of the bias factor is retained for both values of the dislocation density. The positions of the maximum and minimum are shifted towards the smaller sizes of loops with increase in density of sinks (or with decrease in the size of an external toroid). The magnitude of the bias difference between our variant of boundary conditions and the traditional one for both types of loops growth too. 


\section{SUMMARY}

1. Base loops in zirconia are biased sinks, which absorb SIAs more efficiently than vacancies, since $B^{D}, B^{D S}>0$ for both types of loops (see Fig. 4).

2 . There is a good qualitative agreement between the dependence of the bias of loops with $b^{D}=1 / 2[0001]$

$\left(B^{D}\right)$ and $\mathrm{b}^{\mathrm{DS}}=1 / 6<20 \overline{2} 3>\left(B^{D S}\right)$ on their radius. This allows us to assume that the bias of the loop is determined only by the edge component of its Burgers vector.

3. Boundary condition at the outer radius of the toroidal reservoir (9) violates the traditional symmetry in the absorption of PD by loops of different nature, namely: the bias of the vacancy loop depending on its radius has a maximum, while the bias of interstitial loop- a minimum (see Fig. 5).

4. The bias of basic interstitial loops is the smallest, so they can be considered the main sink for vacancies, that makes their existence impossible. This is what is observed in the experiment.

\section{ACKNOWLEDGEMENTS}

This study was performed within the Ukrainian State program Support for the Development of Priority Fields of Scientific Research (KPVK 6541230).

\section{REFERENCES}

1. В.А. Белоус, В.Н. Воеводин, В.И. Змий, Г.Н. Картмазов, С.Д. Лавриненко, И.М. Неклюдов, Н.Н. Пилипенко, Б.А. Шиляев, Б.М. Широков. Современный статус конструкционных материалов ядерных реакторов: Препринт ХФТИ 2013-1. Харьков: ННЦ ХФТИ, 2013, 76 с.

2. M. Griffiths. A review of microstructure evolution in zirconium alloys during irradiation // J. Nucl. Mater. 1988, v. 159, p. 190.
3. C.H. Woo. Defect accumulation behaviour in hcp metals and alloys // J. Nucl. Mater. 2000, v. 276, p. 90.

4. C.H. Woo, U. Gosele. Dislocation bias in an anisotropic diffusive medium and irradiation growth // J. Nucl. Mater. 1983, v. 119, p. 219.

5. C.H. Woo. Theory of irradiation deformation in non-cubic metals: effects of anisotropic diffusion // $\mathrm{J}$. Nucl. Mater. 1988, v. 159, p. 237.

6. G.D. Samolyuk, A.V. Barashev, S.I. Golubov, Y.N. Osetsky, R.E. Stoller. Analysis of the anisotropy of point defect diffusion in hcp $\mathrm{Zr} / /$ Acta Materialia. 2014, v. 78, p. 173.

7. V.I. Dubinko, A.S. Abyzov, A.A. Turkin. Numerical Evaluation of the Dislocation Loop Bias // J. Nucl. Mater. 2005, v. 336, p. 11-21.

8. J.D. Eshelby. The Continuum Theory of Lattice Defects // Solid State Physics. 1956, v. 3, p. 79-144.

9. E. Kröner. Das Fundamentalintegral der anisotropen Differentialgleichungen // Zeitschrift fur Phyzik. 1953, v. 136, p. 402.

10. M.H. Yoo. Elastice interection of small defects and defect clusters in hexagonal crystals // Phys. Stat. Sol. (b). 1974, v. 61, p. 411.

11. P.N. Ostapchuk. Green's Tensor for Crystals of the Hexagonal System // Physics of the Solid State. 2013, v. 55, N 1, p. 100.

12. A.V. Babich, V.F. Klepikov, P.N. Ostapchuk. Bias for the Basal Edge Dislocation Loop in Zirconium: Numerical Analysis // Physics of the Solid State. 2020, v. $62, \mathrm{~N} 12$, p. 2350.

13. L. Fast, J.M. Wills, B. Johansson, O. Eriksson. Elastic constants of hexagonal transition metals: Theory // Phys. Rev. B. 1995, v. 51, p. 17431.

14. A.V. Babich, P.N. Ostapchuk. Bias of basal dislocation loop in zirconium // Problems of Atomic Science and Technology. Series "Physics of Radiation Effect and Radiation Materials Science". 2021, N 2(132), p. 29.

Article received 24.11.2021

\title{
МЕТОД ЛИФШИЦА-РОЗЕНЦВЕЙГА В РАСЧЕТАХ ВIАS-ФАКТОРА БАЗИСНЫХ ДИСЛОКАЦИОННЫХ ПЕТЕЛЬ В ЦИРКОНИИ
}

\author{
О.Г. Троценко, А.В. Бабич, П.Н. Остапчук
}

Методом Лифшица-Розенцвейга получены аналитические выражения для энергии упругого взаимодействия радиационных точечных дефектов дипольного типа с базисными дислокационными петлями циркония: краевой - с вектором Бюргерса $b^{D}=1 / 2[0001]$ и смешанной $-c b^{\text {DS }}=1 / 6<20 \overline{2} 3>$. Они были использованы для численного расчета (методом конечных разностей) bias-фактора этих петель в тороидальном резервуаре с учетом упругой анизотропии гексагонального кристалла. Тороидальная геометрия резервуара позволяет провести расчеты для петли любого размера и без какой-либо коррекции упругого поля в ее области влияния. В приближении центра дилатации получены зависимости bias-фактора петель от их радиуса и природы. Высказывается предположение о том, что bias-фактор определяется только краевой составляющей ее вектора Бюргерса. Показана существенная роль формы граничного условия на внешней поверхности резервуара. 


\section{МЕТОД ЛІФШИЦА-РОЗЕНЦВЕЙГА В РОЗРАХУНКАХ ВІАS-ФАКТОРА БАЗІСНИХ ДИСЛОКАЦІЙНИХ ПЕТЕЛЬ У ЦИРКОНІЇ}

\section{О.Г. Троценко, А.В. Бабіч, П.М. Остапчук}

Методом Ліфшица-Розенцвейга отримані аналітичні вирази для енергії пружної взаємодії радіаційних точкових дефектів дипольного типу з базисними дислокаційними петлями цирконію: крайової - 3 вектором Бюргерса $\mathrm{b}^{\mathrm{D}}=1 / 2[0001]$ та - змішаної $3 \mathrm{~b}^{\mathrm{DS}}=1 / 6<20 \overline{2} 3>$. Вони були використані для чисельного розрахунку (методом кінцевих відмінностей) bias-фактора цих петель у тороїдальному резервуарі 3 урахуванням пружної анізотропії гексагонального кристала. Тороїдальна геометрія резервуару дозволяє проводити розрахунки для петлі будь-якого розміру і без будь-якої корекції пружного поля в області іiі впливу. У наближенні центра ділатації отримані залежності bias-фактора петель від їх радіуса та природи. Висловлюється припущення про те, що bias-фактор визначається тільки крайовою складовою іï вектора Бюргерса. Показана суттєва роль форми граничної умови на зовнішній поверхні резервуара. 